\title{
Forschung zu alpinen Sedimentprozessen als Basis für ein verbessertes Feststoffmanagement unter Berücksichtigung extremer Ereignisse
}

\author{
Marcel Liedermann · Johann Aigner · Andrea Kreisler · Mario Klösch · Rolf Rindler • Philipp Gmeiner • \\ Sebastian Pessenlehner · Michael Tritthart · Christoph Hauer · Helmut Habersack
}

Online publiziert: 24. Januar 2019

(C) Der/die Autor(en) 2019

Zusammenfassung Die Extremhochwässer der Jahre 2002, 2005, 2006, 2009, 2013 und 2018 in Österreich verdeutlichten die Vulnerabilität der Gesellschaft gegenüber derartigen Ereignissen. Die Gewässergeometrie reagiert bei starken Hochwässern mit massiven morphologischen Umlagerungen. Häuser und Infrastruktureinrichtungen in den betroffenen Gebieten sind somit in Gefahr. Daher ist ein verbessertes Verständnis der Sedimenttransportprozesse zentral für die Analyse der Morphodynamik und damit für das Hochwasserrisikomanagement.

Sedimentmonitoring soll ein verbessertes Prozessverständnis bringen, doch beziehen sich bisherige Analysen primär auf die Flussabschnitte, an denen die jeweiligen Daten erhoben wurden. Das Projekt RAISE zielt nun auf die Kombination von Langzeit- mit Kurzzeit-Sedimentforschung (z.B. Extremhochwässer) ab, um diese Daten erstmals in Beziehung zu setzen und integrativ zu diskutieren.

Erste Analysen brachten ein verbessertes Prozessverständnis variabler Transportverhältnisse durch die Definition von Geschiebetransport-Prozesstypen. Diese Klassifizierung hilft in der

DI Dr. M. Liedermann ( $ه)$.

DI J. Aigner · DI A. Kreisler .

DI M. Klösch · DI R. Rindler .

DI P. Gmeiner · DI S. Pessenlehner .

PD DI Dr. M. Tritthart .

PD DI Dr. C. Hauer .

Univ.-Prof. DI Dr. H. Habersack

Department für

Wasser-Atmosphäre-Umwelt,

Christian Doppler Labor

für Sedimentforschung

und -management, Institut

für Wasserbau, Hydraulik

und Fließgewässerforschung,

Universität für Bodenkultur Wien,

Muthgasse 107, 1190 Wien, Österreich

marcel.liedermann@boku.ac.at
Praxis, die Geschiebetransport-Durchfluss-Beziehungen bei variierender Sedimentverfügbarkeit zu verstehen. Die Berechnung des Weitertransports von Geschiebeeinstößen durch das Anwenden einer Transportformel basierend auf Tracerdaten erlaubt die Eingrenzung potenziell von Sohlhebung und kritischer Aufspiegelung betroffener Flussabschnitte während Hochwasserabflüssen. Ein Grundlagenversuch zur Evaluierung der Sammeleffizienz verschiedener Geschiebefänger ermöglichte Erkenntnisse über die Einsatzmöglichkeiten, wie auch über die Einschränkungen der unterschiedlichen Fänger.

Schlüsselwörter Geschiebetransport . Hochwasser .

Sedimenttransportprozesse .

Feststoffmanagement

Research on Alpine sediment processes as a basis for enhanced sediment management, considering extreme events

Abstract Extreme floods in Austria of the years 2002, 2005, 2006, 2009, 2013 and 2018 demonstrate the vulnerability of mankind against such events. River geometry responds to heavy flooding with massive morphological rearrangements. Houses and infrastructure in the affected areas are thus at risk. Therefore, an improved understanding of sediment transport processes is crucial for the analysis of morphodynamics and thus for flood risk management. Sediment monitoring is intended improve process understanding, but analyses so far were focused primarily on the sections of the river where the respective data were collected. The RAISE project now aims to combine long-term and short-term sediment research (such as extreme flood events) in order to relate and integrate this data for the first time.

First analyses provided an improved process understanding of fluctuating transport conditions through the definition of bedload transport process types. This classification helps in practice to understand bedload discharge relationships when having varying sediment availability. The application of a bedload velocity formula is suggested to identify sections which may experience critical bed and related water level changes during floods as a result of sudden bedload supplies from tributaries upstream. A fundamental experiment to evaluate the sampling efficiency of different bedload basket samplers made it possible to gain insights into the possible uses but also about the limitations of the different samplers.

Keywords Bedload transport - Extreme events - Sediment transport processes . Sediment management

\section{Einleitung}

Einhergehend mit der globalen Erwärmung wird auch mit einem Anstieg extremer Wetterbedingungen gerechnet, was $\mathrm{zu}$ verschärften Hochwasserabflüssen in Flusssystemen führen kann (Formayer et al. 2009; IPCC 2012, 2014; Lebensministerium 2012). Internationale Studien belegen diesen dramatischen Anstieg zu Beginn des neuen Jahrtausends, der auch eine Zunahme der Schäden und wirtschaftlichen Verluste zur Folge hat (Barredo 2007). Beispiele für Österreich sind die katastrophalen Hochwasserereignisse von 2002 (9 Menschen getötet, ca. 3200 Mio. EUR Schaden; Habersack et al. 2003), 2005 (3 Personen getötet, ca. 850 Mio. EUR Schaden; Habersack et al. 2009, 2006) und 2013 (5 Menschen getötet, ca. 830 Mio. EUR Schaden). Da die Be- 
völkerung weiter wächst und der Entwicklungsraum in den Alpenregionen sehr begrenzt ist, waren in der Vergangenheit und werden in der Zukunft Siedlungsaktivitäten hauptsächlich entlang der Flusskorridore und in ehemaligen Flussauen (Schober et al. 2015; Habersack et al. 2015) stattfinden und Flussregime und die jeweilige Flussmorphologie weiter stark beeinflussen (De Kok und Grossmann 2010).

Neben den häufig dokumentierten großräumigen Auswirkungen von Überschwemmungen auf die Morphologie eines Flusses ist auch ein detailliertes kurz-, mittel- und langfristiges Prozessverständnis der Morphodynamik erforderlich, da diese in Abhängigkeit des Sedimenttransports auch für die weitere Gefahrenanalyse entscheidend ist. Auch die Veränderung der Verfügbarkeit von Sedimenten aufgrund des Klimawandels, etwa durch Änderungen der Bodennutzung (z. B. erhöhtes Angebot an Feinsedimenten aufgrund von landwirtschaftlicher Bodennutzung und Landentwässerung) oder durch Gletscherschmelze (teilweise erhöhte Sedimentzufuhr) oder Reduktion des Permafrosts hat eine Auswirkung auf die Transportbilanzen. Darüber hinaus kann ein geringerer Abfluss infolge eines wärmeren Klimas auch die Bodenbedeckung verringern, was wieder eher zu einer Erhöhung der Sedimentzufuhr führt (Knox 1983).

Sedimenttransportmechanismen sind derzeit Gegenstand intensiver Forschungsanstrengungen, um die bestehende Wissenslücke hinsichtlich der Vorhersagbarkeit morphodynamischer Änderungen zu schließen. Insbesondere Sedimenttransportprozesse während eines Hochwassers für verschiedene Flusstypen, Seitenerosion und die Vernetzung zu den ökologischen Auswirkungen erfordern noch erheblichen Forschungsaufwand. Das Monitoring von Schlüsselparametern und von $\mathrm{Ge}$ schiebetransportprozessen, die auch für die numerische Modellierung wertvoll sind, zeigt eine große Variabilität der Parameter. Turowski et al. (2011) zeigen, dass die Schwelle für den Bewegungsbeginn eine Streuung im Bereich von einer Größenordnung aufweist und erklärt die Variabilität mit einer starken Abhängigkeit von der hydrologischen Vorgeschichte bei Extremereignissen. Besonders kleine Einzugsgebiete reagieren sehr empfindlich auf Geschiebeeintrag mit einer großen Streuung in den gemessenen Geschiebetranspor- traten (Kreisler et al. 2017; Turowski und Rickenmann 2009).

Der Sedimenttransport wird am Besten durch einen integrativen Monitoringansatz umfassend bestimmt, bei dem mehrere direkte und indirekte Methoden kombiniert werden. Mängel und Einschränkungen einzelner Messmethoden werden damit ausgeglichen. In Österreich werden hochmoderne Messstellen von der Universität für Bodenkultur Wien (BOKU) (Aigner et al. 2017; Habersack et al. 2017; Kreisler et al. 2017; Liedermann et al. 2013) betrieben. Auch die an Österreichs Flüssen angewandte Schwebstoffmonitoringstrategie besteht aus diesen Gründen aus einer Kombination von direkten und indirekten Messmethoden (BMLFUW 2008, 2017; Haimann et al. 2014). Wissenslücken bestehen hier hinsichtlich des detaillierten Prozessverständnisses in Bezug auf Turbulenzwirkungen auf die Partikelbewegung in Abhängigkeit von möglichen Unterschieden in der Einzugsgebietsgeologie und/oder instationärer Strömungshydraulik (Hystereseeffekte), natürlicher Phänomene (wie extreme Hochwässer) oder anthropogene Einwirkungen (wie Hydropeaking bei Wasserkraftanlagen).

In Österreich stehen Daten von 24 Schwebstoff-Messstellen und von 7 von der BOKU mitbetreuten Geschiebemessstellen zur Verfügung. Bisher wurden die Daten dieser Messstellen allerdings primär singulär analysiert. Ein aktuelles Forschungsprojekt mit dem Titel RAISE (Research on Alpine sediment processes, morphodynamics and ecosystem behaviour considering extreme events), gefördert durch die Österreichische Akademie der Wissenschaften (ÖAW), ermöglicht nun eine integrative Analyse der umfangreichen Daten. Durch die Durchführung eines Upscaling-Vorgangs auch mithilfe numerischer Modelle und durch Verwendung der verfügbaren Geschiebeund Schwebstoffdaten aller Messstationen Österreichs wird ein verbessertes Prozessverständnis erwartet. Dieses soll positive Auswirkungen auf ein optimiertes Sedimentmanagement und auch auf die Ökosystemleistung unserer Flüsse haben.

Im ersten Forschungsjahr konnten bereits einige Prozesse besser beschrieben werden, die auch Implikationen auf das Flussgebietsmanagement haben und im Folgenden dargestellt werden.

\section{Methoden}

2.1 Zeitliche und räumliche

Skalenebenen und

Herangehensweise

Basierend auf dem erforderlichen Prozessverständnis, den Wissenslücken und den Schlüsselthemen im Flussgebiets- und Gefahrenmanagement sollen langfristige (z. B. geologische Randbedingungen in einem Einzugsgebiet) mit kurzfristigen Analysen (z. B. Auftreten extremer Überschwemmungen) auf verschiedenen räumlichen Maßstäben kombiniert werden. Die Wechselwirkungen von Prozessen und Problemen im Zusammenhang mit Sedimenten innerhalb eines Flusseinzugsgebiets sind in Abb. 1 dargestellt. Die Messung von Sedimenttransportprozessen liefert dabei wichtige Einblicke in den gesamten Sedimentzyklus und ermöglicht Verknüpfungen zwischen Transportprozessen mit deren Ursachen und Einflussfaktoren.

In der Fachliteratur wird häufig angesprochen, dass die Grundvoraussetzung zur Beantwortung sedimentbezogener Fragen das Vorhandensein von im Freiland erhobenen Daten ist. Diese Voraussetzung ist im Projekt RAISE durch voll ausgestattete Sedimentmonitoringstationen gegeben, mit deren Hilfe der Transportprozess lokal bestimmt wird. Eine Übersicht über die Messstationen geben Kreisler et al. (2014) und Haimann et al. (2018). Langzeitdaten liegen für Schwebstoffe (Haimann et al. 2014) sowie für Geschiebe (Aigner et al. 2017; Habersack et al. 2017; Kreisler et al. 2017; Liedermann et al. 2018) für verschiedene Flusstypen vor (auf Punktebene), die vom steilen Gebirgsbach bis zum großen Kiesbettfluss Donau reichen und werden unter besonderer Berücksichtigung extremer Ereignisse analysiert. Darüber hinaus werden Sedimenttransportprozesse und die Einflüsse natürlicher und anthropogener Veränderungen auf der Flussgebietsebene untersucht. Auf der Einzugsgebietsebene werden dann Faktoren bestimmt, die die Flussmorphologie und den Sedimenttransportprozess beeinflussen und somit die Auswirkungen extremer (Hochwasser-)Ereignisse mitbestimmen.

Das verbesserte Verständnis der Auswirkungen kurz- und langfristiger Einflüsse auf die Flussmorphologie und das Sedimentbudget können dazu beitragen, die Planungskriterien für nach- 


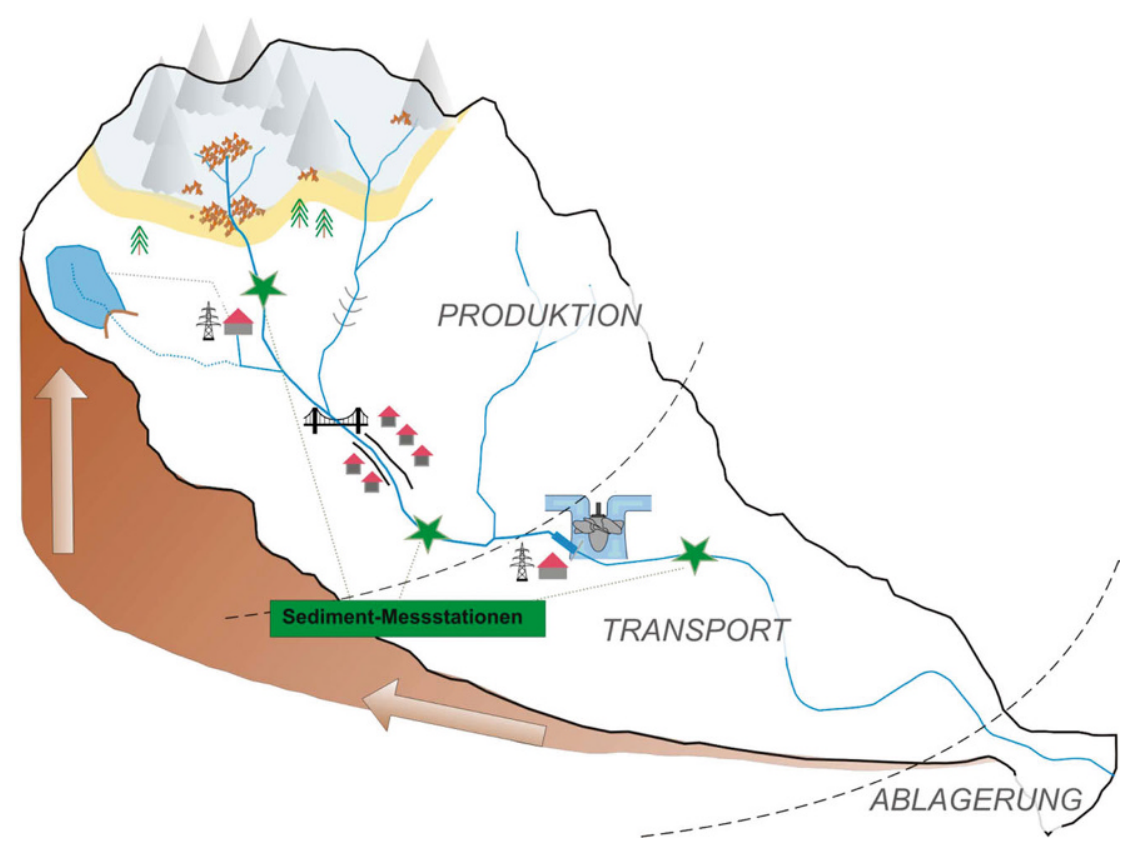

Abb. 1 Sedimentbezogene Prozesse, Einflüsse und Ursachen innerhalb eines Flusseinzugsgebiets

haltige wasserbauliche Maßnahmen (z.B. Hochwasserschutz) oder Flusssanierungsarbeiten und auch die Ökosystemdienstleistungen zu verbessern.

\subsection{Geschiebemessmethoden}

An den meisten Geschiebemessstellen in Österreich werden direkte (Geschiebefänger und Geschiebefalle) und indirekte Messmethoden (Geophonanlage) $\mathrm{zu}$ einer integrativen Geschiebemessstation kombiniert. Die Vernetzung dieser unterschiedlichen Messmethoden erlaubt eine Erfassung von Geschiebetransportraten, -frachten und $\mathrm{Be}$ wegungsbeginn sowie der zeitlichen und räumlichen Variabilität des $\mathrm{Ge}$ schiebetransportprozesses. Eine gute Übersicht der in Österreich verwendeten Methoden ist in Kreisler et al. (2014) und in Habersack et al. (2017) zu finden. An der Messstelle an der Donau werden die Messungen aufgrund der Größe des Flusses bzw. Limitationen durch die Schifffahrt (kein Sohlgurt als Träger einer Geophonanlage möglich) mittels Geschiebefänger, wiederholten Sohlgrundaufnahmen und mit Tracersteinen durchgeführt (Liedermann et al. 2012).

\subsubsection{Mobile Geschiebefangkörbe}

Die Messung mit dem Fänger erfolgt von einem Kranwagen oder Lkw aus, über die gesamte Flussbreite verteilt. Geschiebe, das über die Stahlplatte transportiert wird, erzeugt Vibrationen, die vom Geophon registriert werden. Dieses Signal wird in ein elektrisches Spannungssignal transformiert und von einem Computersystem weiterverarbeitet. Durch die kontinuierliche und automatische Erfassung des Geophonsignals werden zeitlich hoch auflösende Angaben über den Transportprozess gewonnen. Die Bestimmung der transportierten Geschiebemassen ist bei dieser Messmethode nur durch eine Kalibrierung mittels der direkten Messungen möglich. Diese sollten möglichst direkt flussab der Geophone durchgeführt werden. Somit ist auch ein Zusammenhang zwischen Geophondaten und direkt gemessenen Geschiebetransportraten erstellbar.

\subsubsection{Partikeltracer}

An einigen Messstellen werden die Geschiebemessungen durch Tracerstudien ergänzt, bei denen künstlich hergestellte Partikel über längere Zeit im Gebiet verfolgt werden. So können Informationen zur räumlichen und zeitlichen Dynamik des Geschiebetransportprozesses im Einzugsgebiet erlangt werden. Neben aktiven Tracern (Liedermann et al. 2013) kommen auch passive radiotelemetrische Tracer (RFID) zum Einsatz (Rindler et al. 2019, in diesem Heft).

\subsection{Hydrodynamisch-numerische Modellierung}

Eine Geschiebefalle wird möglichst flussab einer Geophonanlage in der Flusssohle eingebaut. Im Ereignisfall kann ein Messschlitz über einen Hydraulikmechanismus geöffnet werden. Das Geschiebe fällt in einen als Falle konzipierten Behälter, der auf Wägezellen gelagert ist. Über die Massenzunahme in der Falle kann der Geschiebetrieb ermittelt werden. Nach einer erfolgreichen Messung wird die Geschiebefalle bei Niederwasser gewartet. Die Textur lässt sich durch Siebung des gesammelten Materials bestimmen. Die Messmethode ermöglicht, vor allem bei Extremereignissen, die Erfassung des Geschiebetriebs.

\subsubsection{Geophonanlage}

Geophone werden auf der Unterseite von Edelstahlplatten an der Gerinnesohle montiert und gleichmäßig
Die Analysen zur Verbesserung des Prozessverständnisses werden durch dreidimensionale, hydrodynamischnumerische Modelle auf großen, mittleren und kleinen Skalenebenen unterstützt. Eine Übersicht über die dabei angewandten Methoden geben Tritthart et al. (2019, in diesem Heft).

\subsection{Forschungsgerinne der BOKU}

Für großskalige Modellversuche, die das Ziel haben, Prozesse unter natürlichen Fließbedingungen $\mathrm{zu}$ untersuchen, besteht seit 2015 das $5 \mathrm{~m}$ breite Forschungsgerinne der BOKU (Abb. 2) zwischen Donau und Donaukanal, welches gleichzeitig die Zuleitung zum zukünftigen neuen Wasserbaulabor darstellt. 


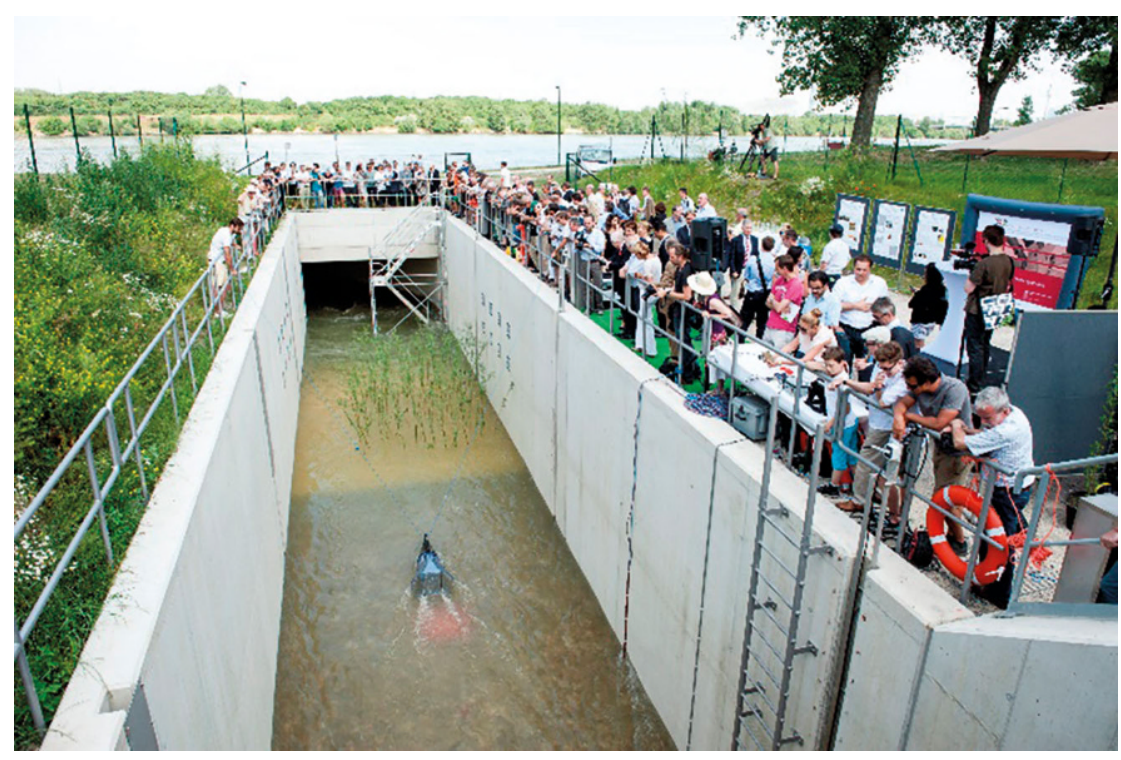

Abb. 2 Forschungsgerinne der BOKU

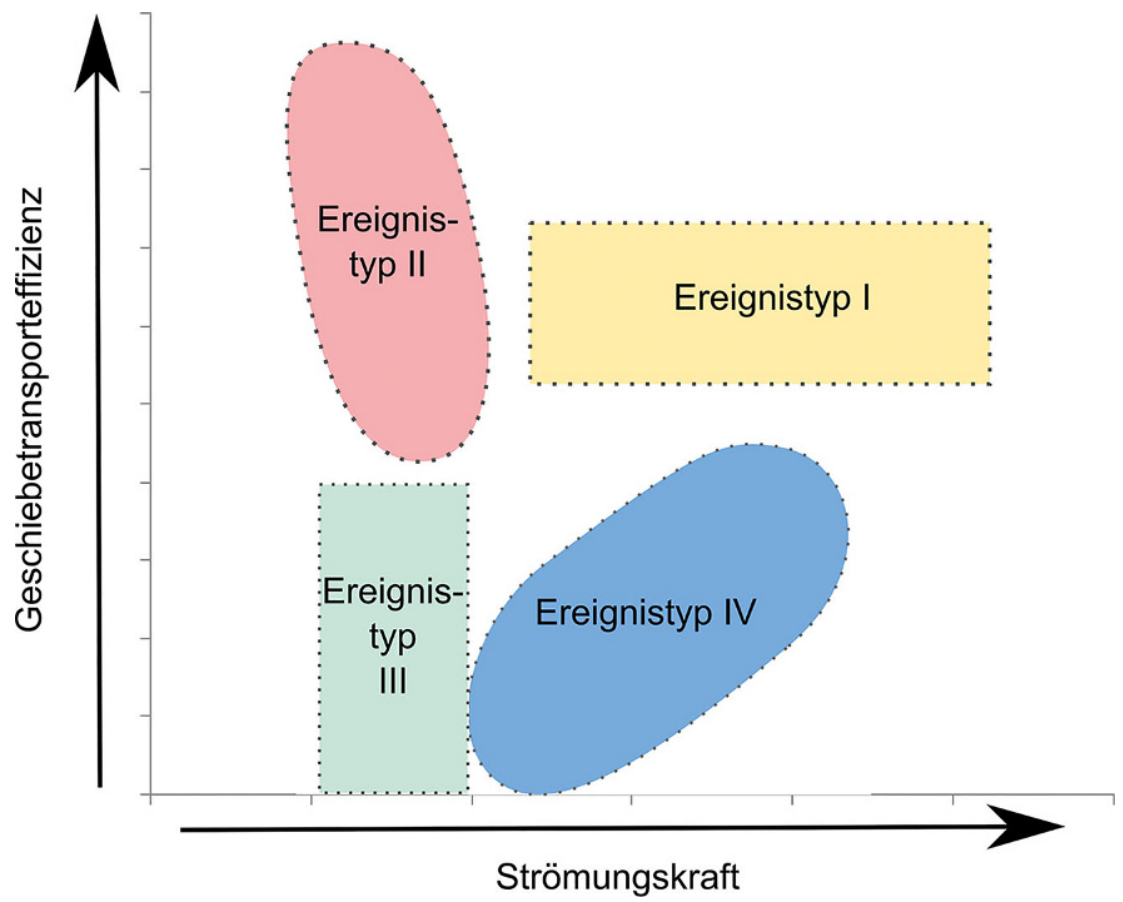

Abb. 3 Konzeptuelle Klassifizierung der Ereignistypen (vereinfacht nach Kreisler et al. 2017)

Bei dieser Forschungsinfrastruktur ist der bis zu $10 \mathrm{~m}^{3} / \mathrm{s}$ einstellbare Freispiegeldurchfluss hervorzuheben. port durch ein integratives Messsystem (Fangkorb, Geschiebefalle und Geophone; erweitert 2017 durch RFID-Tracer) an der Urslau (Salzburg, Maria Alm) gemessen. Die Urslau ist ein Gebirgsfluss, welcher auf etwa $1800 \mathrm{~m}$ Seehöhe entspringt und bis zu seiner Mündung in die Saalach in Saalfelden ein mittleres Gefälle von 5\% und ein Einzugsgebiet von $122 \mathrm{~km}^{2}$ hat. Das Einzugsgebiet weist einen alpinen Charakter auf. Der Sedimenthaushalt der Urslau wird neben Sohl- und Ufererosion stark durch den Input der Zubringer, Mureinstöße und andere Massenbewegungen geprägt (Kreisler et al. 2017).

Ein bestehendes umfassendes Monitoring liefert kontinuierliche Daten des Geschiebetransports in hoher räumlicher und zeitlicher Auflösung und ermöglicht eine Analyse von unterschiedlichen Ereignissen mit Geschiebetransport. Die Messdaten an der Urslau zeigen, dass bei vergleichbaren hydraulischen Verhältnissen unterschiedliche Geschiebemengen transportiert werden können. Die GeschiebetransportDurchfluss-Beziehung an der Urslau weist eine große Bandbreite auf und variiert bei unterschiedlichen Ereignissen.

Basierend auf diesen Beobachtungen wurden 34 Geschiebetransportereignisse (Monitoringjahre 2012 bis 2014) an der Urslau analysiert und klassifiziert (Kreisler et al. 2017). Für jedes Ereignis wurde die mittlere Geschiebetransporteffizienz $e_{p}$ (definiert nach Bagnold 1966; Gl. 1) berechnet und den hydraulischen Verhältnissen gegenübergestellt:

$$
e_{p}=\frac{I_{b} \tan \alpha}{\Omega_{\mathrm{ex}}}
$$

Darin bedeutet $I_{b}$ die Geschiebemenge unter Wasser $\left[\mathrm{m}^{3}\right]$, die über Gl. 2 berechnet wird,

$$
\left.I_{b}=Q_{B v} g \rho_{s}-\rho\right)
$$

mit $Q_{B v}$ als volumetrische Geschiebefracht während eines Ereignisses [ $\left.\mathrm{m}^{3}\right], g$ als Erdbeschleunigung, $\rho_{s}$ als Feststoffdichte und $\rho$ als Fluiddichte. $\alpha$ in Gl. 1 ist der innere Reibungswinkel des Sediments und wurde, wie in Recking (2012) vorgeschlagen, mit $52^{\circ}$ angenommen. Die vorhandene Strömungsenergie $\Omega_{e x}$ berechnet sich durch

$$
\Omega_{\mathrm{ex}}=\mathrm{V}_{\mathrm{ex}} \rho \mathrm{gS}
$$

Seit 2011 wird im Auftrag - und in intensiver Zusammenarbeit mit - der Wildbach- und Lawinenverbauung vom IWA/BOKU der Geschiebetrans- 


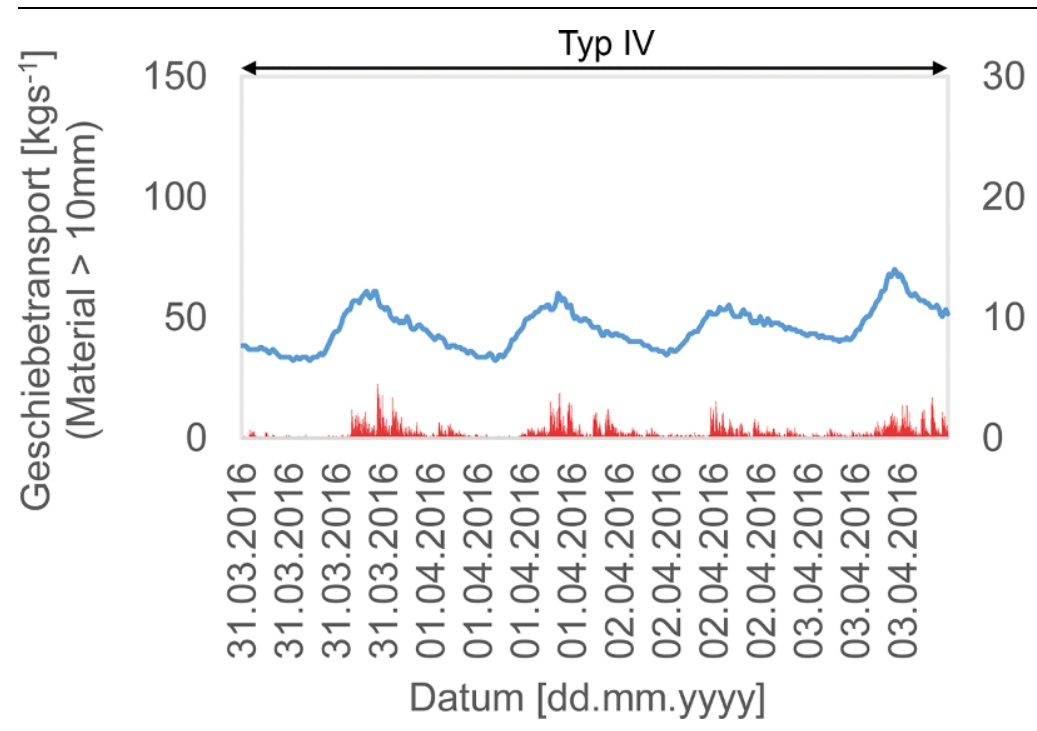

Abb. 4 Beispiel für Ereignistyp IV: Durchfluss- und Geschiebetransportganglinie (D) $10 \mathrm{~mm}$ ) während der Schneeschmelze (31.03.2016 bis 03.04.2016)

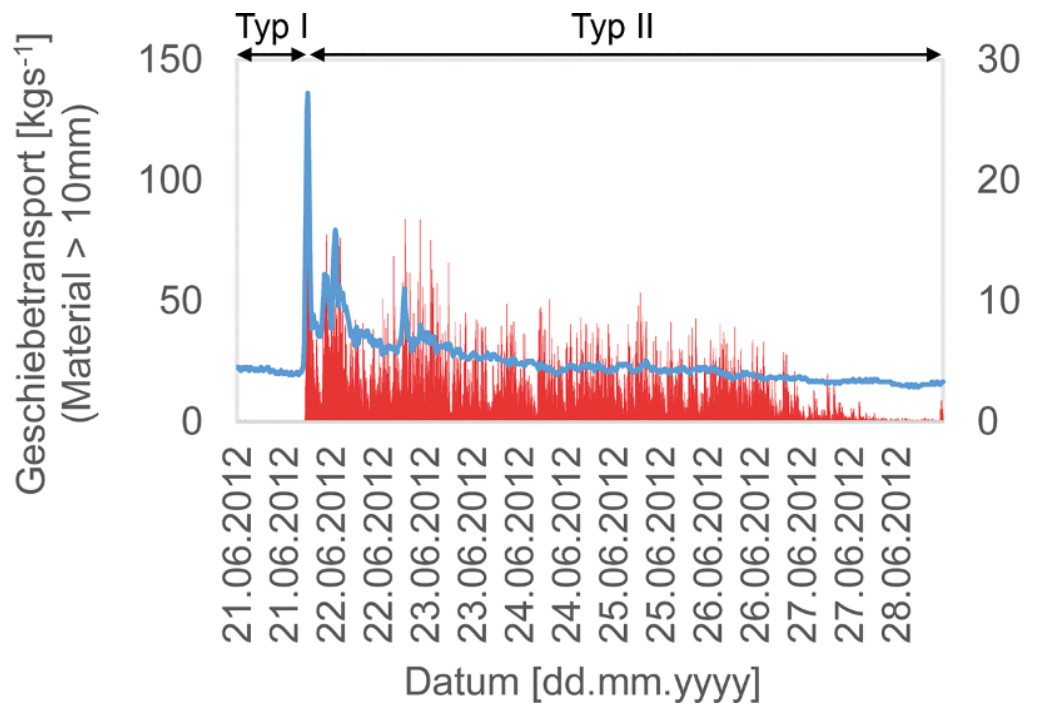

Abb. 5 Beispiel für Ereignistyp I und II: Durchfluss- und Geschiebetransportganglinie (D>10 mm) während eines Sommerereignisses (21.06.2012 bis 28.06.2012)

mit $V_{e x}$ als abflusswirksame Durchflussfracht während eines Ereignisses und $S$ als dem Sohlgefälle.

Diese Gegenüberstellung führt $\mathrm{zu}$ einer Klassifizierung der Geschiebetransportereignisse in 4 Typen: Typ I beschreibt jene Ereignisse, wo bei hohem Durchfluss eine große Geschiebemenge mobilisiert und transportiert wird; bei Typ II handelt es sich um Folgeereignisse von Typ I. Hier wird Material, welches bei Typ I mobilisiert wurde und in großen Mengen verfügbar ist, bei vergleichsweise geringen Durchflüssen transportiert. Unter Typ III werden vor allem kleine, häufig auftretende
Ereignisse zusammengefasst. Bei diesen Ereignissen werden leicht mobilisierbare Sedimente aus der Flusssohle transportiert. Geschiebeereignisse, bei denen wenig Material vorhanden ist (z.B. während der Schneeschmelze), werden in Typ IV zusammengefasst. Die Anordnung der Ereignistypen in Bezug auf die Strömungskraft (stream power) und die Geschiebetransporteffizienz ist in Abb. 3 zu sehen.

Beispiele für Geschiebetransportereignisse, die den beschriebenen Ereignistypen zugeordnet werden können, sind in Abb. 4 und 5 zu finden.
Durch die Zuordnung der Geschiebetransportereignisse $\mathrm{zu}$ den unterschiedlichen Ereignistypen können transportbeeinflussende Faktoren besser berücksichtigt und die Beziehung zwischen Durchfluss und Geschiebetransport für die jeweiligen Ereignistypen getrennt erstellt werden. Sedimentbilanzen und Transportspitzen während Extremereignissen können so besser bestimmt werden.

\subsection{Auswirkungen der Sedimentverfügbarkeit auf den Geschiebetransport}

Neben der Gerinnegeometrie sind die wesentlichen Einflussfaktoren auf den Geschiebetransport die hydraulischen Bedingungen (Wasserstand/Durchfluss) sowie die Sedimentverfügbarkeit (Korngrößenverteilung, Geschiebeinput). Ein großer Teil der derzeit in der Praxis verwendeten Geschiebetransportformeln wurde in Laborversuchen mit konstanter Geschiebezugabe und klar definierter Modellgeometrie entwickelt. Bei der Anwendung dieser Transportformeln wird daher meist nur die Hydraulik als veränderliche Größe betrachtet, wodurch erhöhter Geschiebetransport einzig über einen gesteigerten Durchfluss erzielt werden kann. Naturmessungen des Geschiebetransports weisen jedoch, auch bei stationären Durchflussverhältnissen, oftmals eine ausgeprägte zeitliche Variabilität (pulsierender Geschiebetransport) auf. Darüber hinaus konnte vor allem an kleineren Flüssen beobachtet werden, dass der größte Geschiebetransport nicht am Durchflussmaximum auftritt. Grund dafür ist meist eine Veränderung in der Sedimentverfügbarkeit im Fluss. Ein markantes Beispiel hierfür ist in Abb. 6 dargestellt und zeigt Durchfluss und Geschiebetransport an der Geschiebemessstelle Lienz/Drau beim Durchgang eines Hochwasserereignisses $\left(\mathrm{HQ}_{1}\right.$ bis $\mathrm{HQ}_{2}$ ).

Während des Hochwasserereignisses wurden über Zubringer markante Mengen an Sedimenten in die Restwasserstrecke eingetragen. Dieser sprunghafte Anstieg an transportierbarem Flusssediment wird Geschiebewelle oder Geschiebepuls genannt. Da Geschiebepartikel eine deutlich geringere Transportgeschwindigkeit als Wasser aufweisen, wird das neu eingetragene Flusssediment zeitlich verzögert flussab transportiert. Dies führt zu ausgeprägten Hystereseeffekten zwischen Durchfluss 


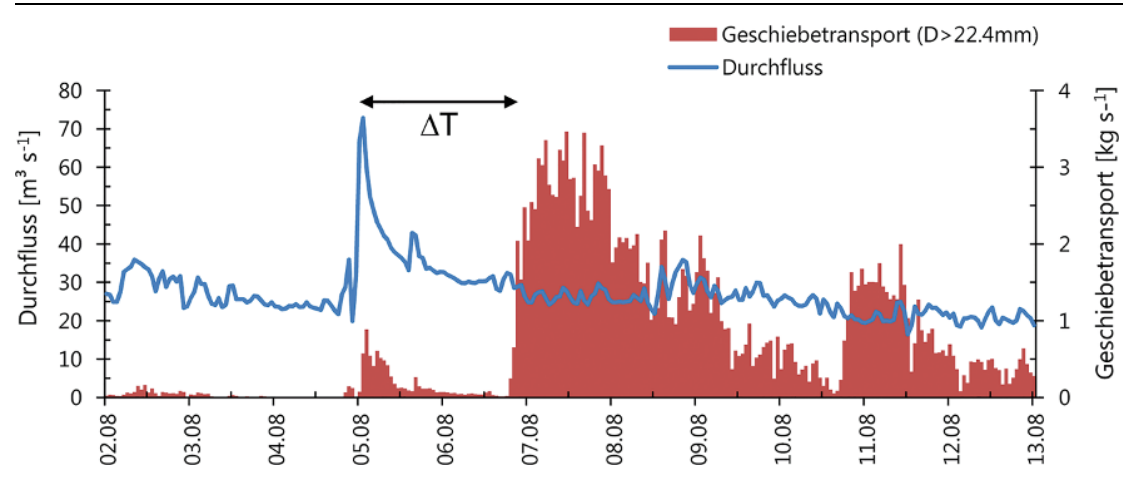

Abb. 6 Ganglinien von Durchfluss (blau) und Geschiebetransport (rot) an der Geschiebemessstelle Lienz/Drau im Zeitraum 02.-13.08.2015. Sedimenteintrag aus der Restwasserstrecke führt zu einem zeitlich versetzten Anstieg des Geschiebetransports (adaptiert nach Aigner et al. 2017)

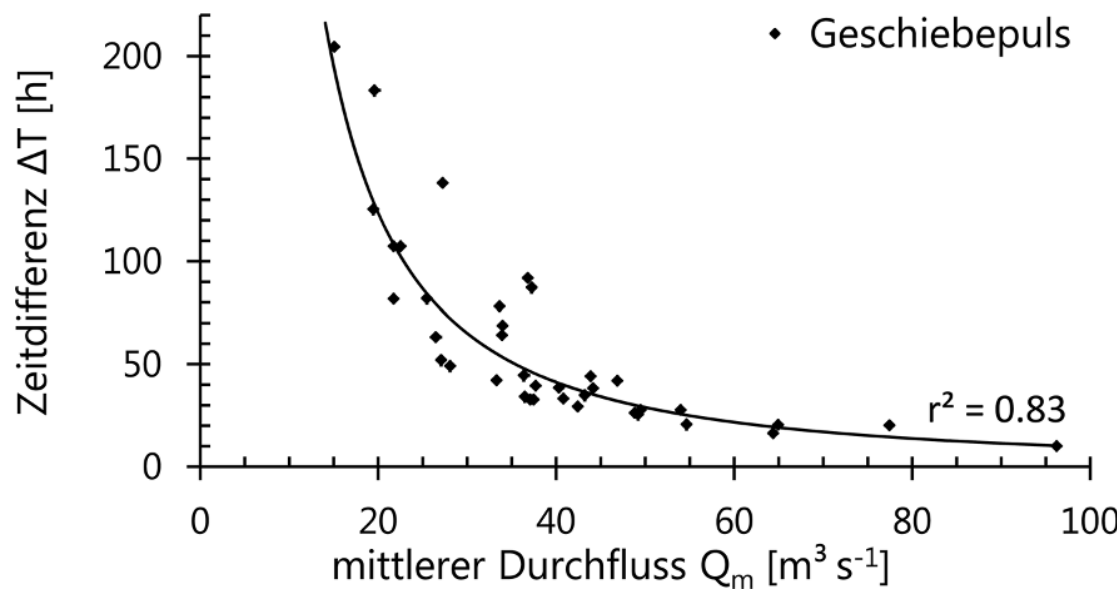

Abb. 7 Laufzeit von 38 Geschiebepulsen in Abhängigkeit vom mittleren Durchfluss an der Geschiebemessstation Lienz/Drau im Zeitraum 2001 bis 2015. Je größer und länger ein Durchflussereignis ist, desto schneller wird ein Geschiebepuls flussab transportiert (adaptiert nach Aigner et al. 2017)

und Geschiebetransport. Der durch das Hochwasserereignis eingetragene Geschiebepuls (siehe Abb. 6) erreichte beispielsweise erst knapp zwei Tage nach dem Durchflussmaximum die Geschiebemessstation. Der Geschiebepuls umfasste ein Volumen von etwa $550 \mathrm{~m}^{3}$ und führte auch Wochen nach dem Ereignis noch zu einer deutlichen Erhöhung des Geschiebetransports an der Station.

Das natürliche Geschiebekontinuum flussauf der Messstation Lienz/Drau ist stark durch eine $24 \mathrm{~km}$ lange Restwasserstrecke beeinflusst. Durch die anthropogen veränderten Durchflussbedingungen in der Restwasserstrecke findet eine Mobilisierung von Geschiebematerial daher fast ausschließlich während Hochwasserereignissen statt. Seit Errichtung der Geschiebemessstation im Jahre 2001 konnten über passierten. Aigner et al. (2017) zeigten, dass die Zeitdifferenz bis zur Ankunft der Geschiebepulse maßgeblich vom mittleren Durchfluss nach dem ersten markanten Durchflussanstieg abhängt (Abb. 7). Dieser Zusammenhang erlaubt eine Prognose über die Ankunft von Geschiebepulsen an der Station allein aufgrund der Durchflussentwicklung. Für die 38 beobachteten Geschiebepulse konnte eine Transportgeschwindigkeit von minimal 0,002 bis $0,05 \mathrm{~m} \mathrm{~s}^{-1}$ berechnet werden.

Die Entstehung von Geschiebepulsen ist nicht auf anthropogen beeinträchtigte Flüsse beschränkt, sondern kann auch in weitgehend natürlichen Einzugsgebieten beobachtet werden. Die oft sehr schnellen Änderungen in der Geschiebeverfügbarkeit führen, bei gleichen Durchflussverhältnissen, zu einer großen Bandbreite an möglichen Geschiebetransportraten. Die Geschiebemesssdaten der letzten Jahre weisen darauf hin, dass kleinere Einzugsgebiete (z.B. Rofenache/Tirol) deutlich schneller und markanter auf Änderungen im Geschiebekontinuum reagieren. Größere Flusssysteme (z.B. Drau, Donau) können aufgrund ihrer Ausdehnung Änderungen in der Sedimentverfügbarkeit in gewissem Ausmaß puffern und dämpfen. Für eine qualitativ gute $\mathrm{Ab}$ schätzung des Geschiebehaushalts kleinerer Flüsse und Einzugsgebiete sind daher indirekte Geschiebemessmethoden (z.B. Geophone) zu empfehlen.

\subsection{Transportgeschwindigkeiten von Tracersteinen als Basis einer Analyse des Transfers von Geschiebeeinstößen}

Eine im Rahmen des EU Interreg Alpine Space-Projekts „HyMoCARES“ durchgeführte Studie mit künstlichen Steinen an der Drau, die mit aktiv sendenden Radiotransmittern ausgestattet wurden, diente der Untersuchung der Transportgeschwindigkeit des Geschiebes zum besseren Verständnis des Sedimenttransfers in Flüssen. Gl. 4 konnte anhand einer Weiterentwicklung der Regressionsmethode von Klösch und Habersack (2018) von den Monitoringdaten abgeleitet werden (Klösch et al., in Vorbereitung):

$$
\begin{aligned}
V_{u}= & 0,21 e^{3,63\left(\frac{d}{d_{50}}\right)} \sqrt{\frac{\rho_{s}-\rho}{\rho} g d} \\
& {\left[\frac{\tau}{\left(\rho_{s}-\rho\right) g d}-0,021\left(\frac{d}{d_{50}}\right)^{-0,485}\right]^{\frac{3}{2}}, }
\end{aligned}
$$

worin $V_{u}$ die virtuelle Transportgeschwindigkeit bei der jeweiligen Sohlschubspannung (Ruhephasen eingerechnet) darstellt, $e$ die Eulersche Zahl, $d$ die b-Achsenlänge des transportierten Sediments, $d_{50}$ der Median der b-Achsenlänge des Sediments an der Sohlenoberfläche, bestimmt anhand der Pebble-Count-Methode nach Wolman (1954), $\varrho_{\mathrm{s}}$ die spezifische Dichte des Sediments, $\varrho$ die Dichte von Wasser, $g$ die Erdbeschleunigung und $\tau$ die Sohlschubspannung (basierend auf dem Mittelwert zwischen mittlerer und maximaler Wassertiefe in Querprofilen; Ferguson und Wathen 1998). Dabei ist $\mathrm{zu}$ erwähnen, dass im Zeitraum der Messungen kein Aufbrechen der 
Hier steht eine Anzeige.

Springer 
Hier steht eine Anzeige.

Springer 


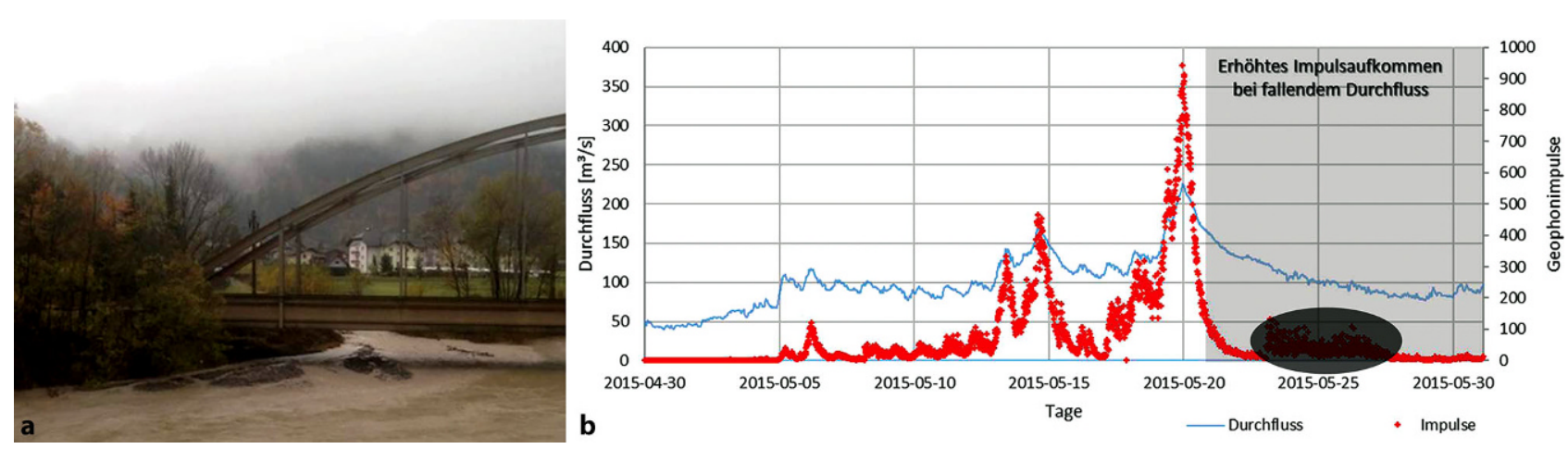

Abb. 8 a Geschiebeeinstoß am 06.11.2014 aus dem Gailbergbach in die Drau (4,5 km flussauf der Geschiebemessstation bei Dellach), b Anomalien in der Relation zwischen Geophonimpulssignalen und Durchfluss im darauffolgenden Frühjahr an der flussab liegenden Geschiebemessstation bei Dellach

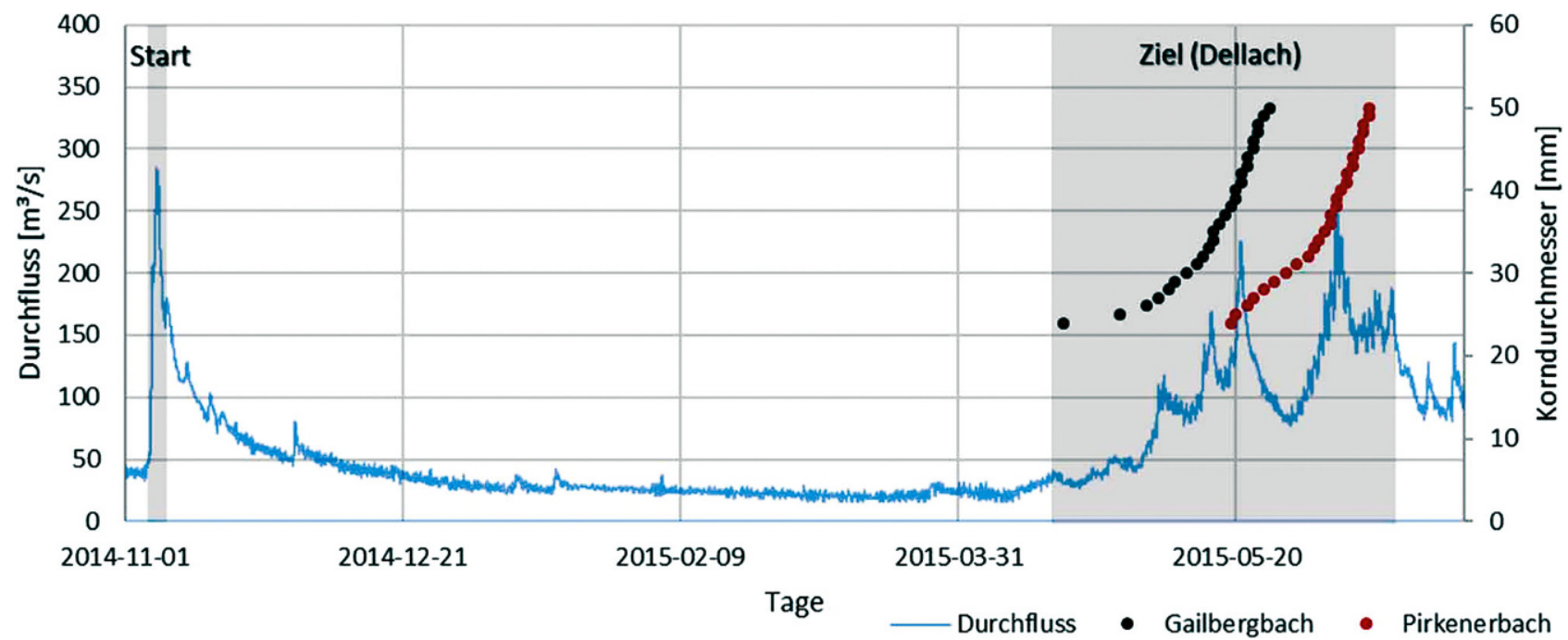

Abb. 9 Ankunft der verschiedenen Korndurchmesser an der Geschiebemessstation gemäß der Geschiebegeschwindigkeit nach Gl. 4: Die Berechnungen starteten an den Mündungen der Zubringerbäche Gailbergbach und Pirkenerbach zum Zeitpunkt der Geschiebeeinstöße

Deckschicht stattfand und daher wahrscheinlich Geschiebe über eine intakte Deckschichte transportiert wurde.

Die abgeleitete Geschiebegeschwindigkeitsformel (Gl. 4) wurde im weiteren Schritt dazu verwendet, die Transportdauer von Geschiebeeinstößen aus Zubringern bis zur Geschiebemessstation in Dellach zu berechnen. Die berechnete Ankunftszeit des Geschiebeeinstoßes wurde folgend den Geophondaten der Geschiebemessstation gegenübergestellt. Dazu wurden die an der Geschiebemessstation an der Drau in Dellach aufgezeichneten Geophonsignale dezidiert auf Abweichungen in der Transportintensität untersucht. Im Mai 2015 fand beispielsweise ein solches vermehrtes Geschiebetransportaufkommen statt, das von der normalerweise relativ stabilen Be- ziehung zwischen Geschiebeimpulssignalen und Durchfluss an der Station abwich. Beobachtete Geschiebeeinstöße in die Drau aus Zubringern, die $4,5 \mathrm{~km}$ bzw. $7,5 \mathrm{~km}$ flussauf in die Drau münden, wurden als Ursache für diese Anomalien vermutet (Abb. 8).

Eine Berechnung der Transportweiten zwischen dem Zeitpunkt des Geschiebeeinstoßes und dem Zeitpunkt der erhöhten Transportintensität an der Geschiebemessstation anhand von Gl. 4 lässt den Schluss zu, dass die Messung des erhöhten Transports auf den Eintrag dieser Zubringer zurückzuführen ist. Abb. 9 zeigt, in welchem Zeitraum die unterschiedlichen Kornfraktionen gemäß Gl. 4 an der Geschiebemessstation eingelangt sein müssten. Dieser Zeitraum deckt sich mit dem Zeitraum der gemessenen Anomalie.
Die Geophonimpulse und die Ergebnisse der Traceruntersuchungen können in weiterer Folge auch der Untersuchung der Diffusion des eingetragenen Geschiebes dienen (insbesondere in longitudinaler Richtung; Abb. 10), womit sich der Effekt des Geschiebeeintrags auf Sohlhöhenänderungen abschätzen ließe.

\subsection{Grundlagenversuch zur Sammeleffizienz von Geschiebefangkörben im Forschungsgerinne der BOKU}

Zurzeit gibt es in Österreich sieben Messstellen, an denen die Messungen mit verschiedenen Geschiebefangkörben durchgeführt werden. Dabei werden primär drei verschiedene Bautypen verwendet: Large Helley Smith Samp- 


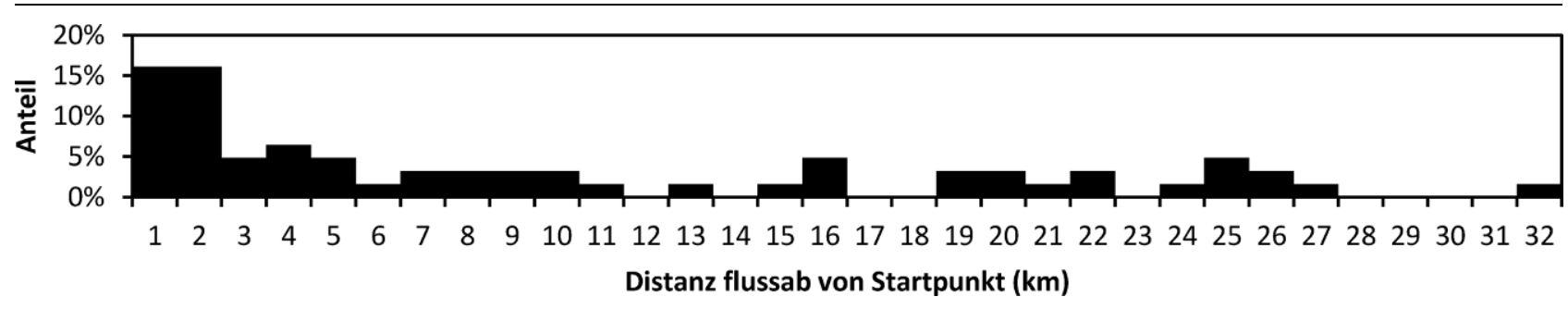

Abb. 10 Verteilung der Tracer durch Transport und Diffusion nach dem Einsatz bei der Ortschaft Irschen vom 11. Mai 2017 bis zum 31. August 2017. Die Korngrößen der Tracer liegen zwischen 32 und $140 \mathrm{~mm}$ (b-Achsenlänge)

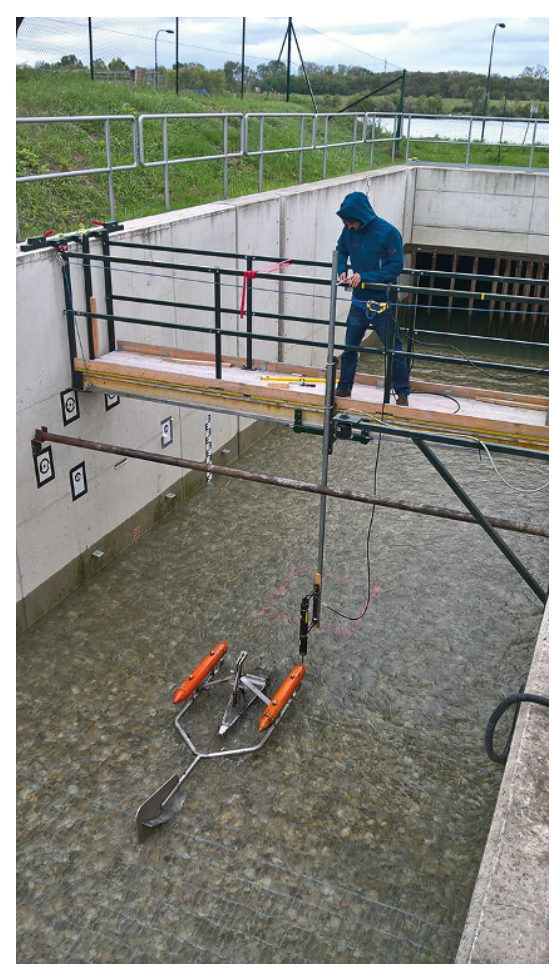

Abb. 11 Versuchssetup für die Analyse der Sammeleffizienz des BfG-Sammlers im Forschungsgerinne

ler, Vent-Sammler und BfG-Sammler. In Laborversuchen wurden alle drei verwendeten Geschiebesammler bereits evaluiert, in der Natur gibt es aber Randbedingungen, die die Sammeleffizienz beeinflussen können, die unter Laborbedingungen nicht eingestellt werden können (z. B. Turbulenzschwankungen, kohärente Strukturen). Weiters liegt an jeder Messstelle eine andere Ausgangssituation vor, und es ist nicht immer eindeutig, welcher Sammler für die Beprobung vor Ort herangezogen werden soll. Deshalb wurden die drei Fängertypen im Forschungsgerinne einem naturnahen Geschiebetrieb ausgesetzt, um die Unterschiede in der Sammeleffizienz herauszuarbeiten. Das
Versuchssetup ist beispielhaft für den BfG-Sammler in Abb. 11 dargestellt.

Die Versuche im Forschungsgerinne liefern die Grundlage, um die Einsatzmöglichkeiten der unterschiedlichen Sammler zu optimieren. Zum Beispiel zeigte sich, dass der Vent-Sammler erst bei hoher hydraulischer Belastung eine gute Sammeleffizienz aufweist. Bei den Strömungsbedingungen im Forschungsgerinne konnte die gewünschte hydraulische Effizienz primär aufgrund der Netze nicht erreicht werden (Abb. 12)

Aufgrund seiner Robustheit, der einfachen Handhabung und dem hohen potenziellen Sammelvolumen ist der Vent-Sammler an einigen österreichischen Messstellen essenziell wichtig. Vor allem an alpinen Flüssen mit hohen Geschiebetransportraten ist der Einsatz anderer Geschiebesammler meist nicht möglich. Aufgrund der eingeschränkten Sammeleffizienz bei geringen Fließgeschwindigkeiten wird der Vent-Sammler in Österreich allerdings nur an Messstellen mit sehr hohen Fließgeschwindigkeiten verwendet. Aufgrund der oft sehr hohen Transportraten verkürzt sich hier die Messdauer oftmals auf wenige Sekunden, wodurch die hydraulische Effizienz im Vergleich zu anderen potenziellen Messfehlern (Messzeit, schnelle Positionierung auf der Sohle, korrekte Ausrichtung in Fließrichtung etc.) nur mehr untergeordneten Einfluss auf das Sammelergebnis hat. Bei Flüssen mit niedrigen Fließgeschwindigkeiten und geringen Geschiebetransportraten ist aufgrund der meist deutlich längeren Messdauer - die hydraulische Effizienz von größerer Bedeutung, wodurch hier der Einsatz von Druckdifferenzsammlern (LHS, BFG) zu empfehlen ist.
4 Schlussfolgerungen im Zusammenhang mit positiven Managementimplikationen durch das verbesserte Prozessverständnis

Die vorgestellte Klassifizierung der Geschiebetransportereignisse stellt für die Praxis ein wichtiges Werkzeug dar, um die Geschiebetransport-Durchfluss-Beziehungen an einem Gebirgsfluss mit variierender Sedimentverfügbarkeit zu verstehen. Stark fluktuierende Ergebnisse für den Sedimenttransport - speziell bei Extremereignissen - können so richtig eingeordnet werden und die Jahresfrachten basierend auf punktuellen Messungen können durch das verbesserte Prozessverständnis genauer bestimmt werden.

Die Analyse der Laufzeit von Geschiebepulsen aus der Restwasserstrecke an der Drau/Lienz führte zu einer deutlich verbesserten Planung direkter Geschiebemessungen, vor allem bei sehr hohen Transportintensitäten. Das Prozessverständnis über Entstehung und Transport von Geschiebepulsen kann zukünftig für ein verbessertes Geschiebemanagement der Restwasserstrecke an der Oberen Drau genutzt werden. Da sich Veränderungen in der Sedimentverfügbarkeit, zeitlich versetzt, in Änderungen der Geschiebetransportraten auswirken, kann der Einfluss von Maßnahmen in der Restwasserstrecke (z.B. Aktivierung von Aufweitungen) erfasst und quantifiziert werden. In Kombination mit Geschiebetracern ist in Zukunft durch die Analyse der Laufzeiten von Geschiebepulsen sogar die Lokalisierung von Geschiebequellen denkbar.

Während Hochwässern und insbesondere bei Extremereignissen kann es zu Änderungen der Sohlhöhe und der Morphologie sowie zur Entstehung oder Veränderung von Bettstrukturen mit Auswirkungen auf die Wasserspiegellagen kommen. Dabei können Verbreiterung von bis zur 14-fachen Breite 

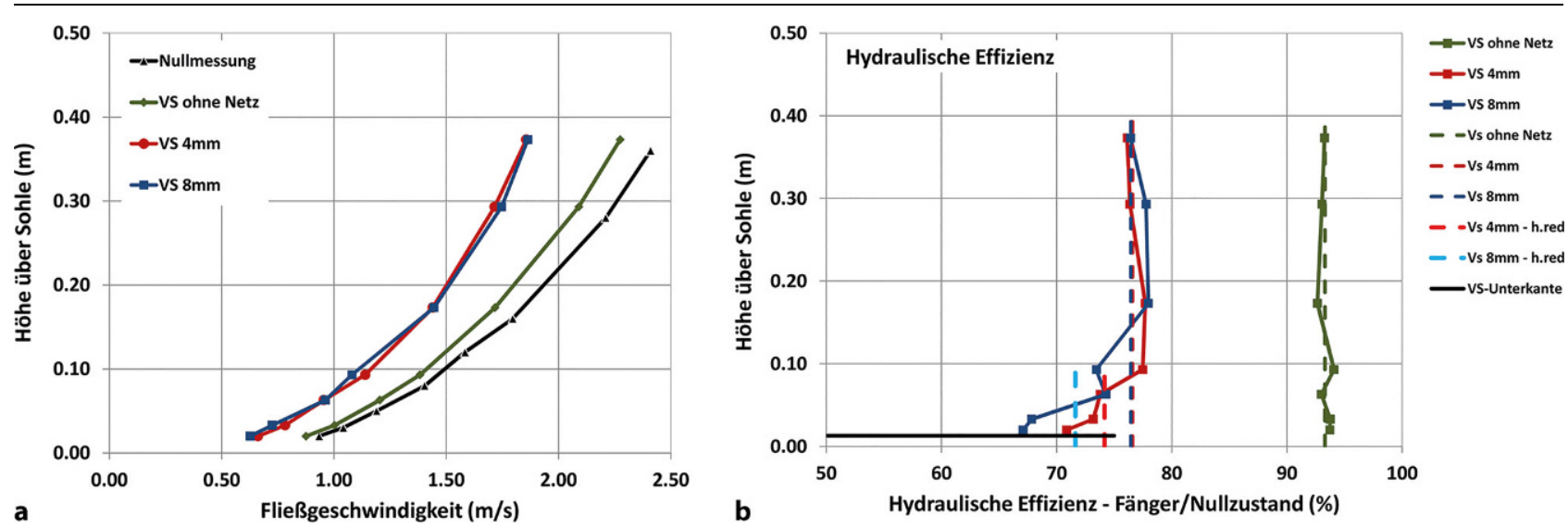

Abb. 12 a Vergleich der Fließgeschwindigkeiten: Nullmessung (ohne Einbau eines Sammlers) und Vent-Sammler (VS) mit unterschiedlichen Netztypen und ohne Netz; b Vergleich der hydraulischen Effizienz des Vent-Sammlers (VS) mit unterschiedlichen Netztypen und ohne Netz

auftreten (Hauer und Habersack 2009; Krapesch et al. 2011). Geschiebeeinstöße von Zubringern können durch den vermehrten Eintrag von Sediment dabei eine wesentliche Rolle spielen. In kritischen Abschnitten (z. B. Abschnitte mit hohem Schadenspotenzial bei gleichzeitig beobachteten Anlandungstendenzen) könnte dadurch der Hochwasserschutz stark beeinträchtigt sein. Das Wissen über die Geschwindigkeit und Diffusion des Geschiebes, gemeinsam mit der Messung oder Berechnung der transportierten Menge, erlaubt eine Abschätzung der möglichen Sohlhöhenänderungen flussab der Geschiebeeinstöße und außerdem eine Eingrenzung potenziell betroffener Flussabschnitte während Hochwasserabflüssen. Trifft das Geschiebe gemäß den Berechnungen zum Zeitpunkt der Spitzenabflüsse in kritischen Flussabschnitten ein, sind dort prioritär Maßnahmen zu setzen, um die Abflusskapazität zu erhöhen oder um Raum für sich ablagerndes Material zu schaffen. Auch für die Berechnung von Hochwasserspiegellagen und bei der Planung von Hochwasserschutzmaßnahmen ist die Diffusion der Geschiebeeinstöße eine wertvolle Zusatzinformation.

Das Wissen über die Einsatzmöglichkeiten, aber auch über die Einschränkungen der unterschiedlichen Geschiebefänger, erlaubt deren gezielten Einsatz und garantiert gut interpretierbare Ergebnisse im Zuge von Geschiebetransportmessungen.

Insgesamt sind die Messungen aber primär auch die hier durchgeführten weiterführenden Analysen auf unterschiedlichen Skalenebenen - wichtige Schritte, um die Sedimenttransportprozesse besser verstehen zu können und damit das Feststoffmanagement an alpinen Flüssen zu verbessern.

Danksagung Das Projekt RAISE wird durch die Österreichische Akademie der Wissenschaften (ÖAW) über die Forschungsinitiative „Earth System Sciences" (ESS) finanziert. Weiters danken wir für die finanzielle Unterstützung durch das Bundesministerium für
Digitalisierung und Wirtschaftsstandort und die Nationalstiftung für Forschung, Technologie und Entwicklung. Dank gebührt außerdem Stephan Schober von der Kärntner Landesregierung für die Hinweise auf Herausforderungen in der Praxis und die fachliche Diskussion.

Funding Open access funding provided by University of Natural Resources and Life Sciences Vienna (BOKU).

Open Access Dieser Artikel wird unter der Creative Commons Namensnennung 4.0 International Lizenz (http:// creativecommons.org/licenses/by/4. $0 /$ deed.de) veröffentlicht, welche die Nutzung, Vervielfältigung, Bearbeitung, Verbreitung und Wiedergabe in jeglichem Medium und Format erlaubt, sofern Sie den/die ursprünglichen $\mathrm{Au}$ tor(en) und die Quelle ordnungsgemäß nennen, einen Link zur Creative Commons Lizenz beifügen und angeben, ob Änderungen vorgenommen wurden.

\section{Literatur}

Aigner, J, Kreisler, A., Rindler, R., Hauer, C., Habersack, H. (2017): Bedload pulses in a hydropower affected alpine gravel bed river GEOMORPHOLOGY. 2017; 291: 116-127.

Bagnold, R.A. (1966): An Approach to the Sediment Transport Problem from General Pyhsics. Geological survey Professional Paper, United States Government Printing Office, Washington, D.C.

Barredo, J. I. (2007): Major flood disasters in Europe: 1950-2005. Journal of Natural Hazards 42, 125-148.

BMLFUW (2008): Feststoffe im Fließgewässer Leitfaden zur Erfassung des Schwebstofftrans- portes. Bundesministerium für Land- und Forstwirtschaft, Umwelt und Wasserwirtschaft, Wien. BMLFUW (2017): Schwebstoffe im Fließgewässer - Leitfaden zur Erfassung des Schwebstofftransports. Bundesministerium für Land- und Forstwirtschaft, Umwelt und Wasserwirtschaft, 2. Auflage, Wien.

Ferguson, R. I., Wathen, S. J. (1998): Tracer-pebble movement along a concave river profile: virtual velocity in relation to grain size and shear stress. Water Resources Research 34: 2031-2038. Formayer, H., Kromp-Kolb, H., Schwarzl, I. (2009): Auswirkungen des Klimawandels auf Hochwasserereignisse in Oberösterreich (Endbericht Band 2 der Forschungsreihe „Auswir- kungen des Klimawandels auf Oberösterreich“, Mai 2007). BOKU-Met Report, 14

Habersack, H., Bürgel, J., Kanonier, A. (2009): FloodRisk II - Vertiefung und Vernetzung zukunftsweisender Umsetzungsstrategien zum integrierten Hochwassermanagement.

Habersack, H., Fuchs, H., Sattler, S., Wind, H. (2003): Hochwasser 2002 - Datenbasis der Schadensbilanz 2002 (Flood 2002 - data basis of damage records 2002): StartClim.9. ZENAR.

Habersack, H., Kreisler, A., Rindler, R., Aigner, J., Seitz, H., Liedermann, M., Laronne, J. B. (2017): Integrated automatic and continuous bedload monitoring in gravel bed rivers GEOMORPHOLOGY. 2017; 291: 80-93. 
Habersack, H., Rudolf-Miklau, F., Godina, R., Krapesch, G. (2006): Hochwasser 2005 - Ereignisdokumentation (Flood 2005 - documentation of the flood event). Lebensministerium (ed.), Vienna.

Habersack, H., Schober, B., Hauer, C. (2015): Floodplain evaluation matrix (FEM): An interdisciplinary method for evaluating river floodplains in the context of integrated flood risk management. NAT HAZARDS. 2015; 75: S5-S32

Haimann, M., Aigner, J., Gmeiner, P., Lalk, P., Habersack, H. (2018): Hochwasser und Feststoffe: vom Sedimenttransport zum flussmorphologischen Raumbedarf. Österreichische Wasserund Abfallwirtschaft, 70, 78-89; ISSN 0945-358X Haimann, M., Liedermann M., Lalk P., Habersack H. (2014): Development and evaluation of an integrated suspended sediment transport monitoring and analysis concept. International Journal of Sediment Research 29(2): 135-148

Hauer, C., Habersack, H. (2009): Morphodynamics of a 1000-year flood in the Kamp River, Austria, and impacts on floodplain morphology EARTH SURF PROCESS LANDF. 2009; 34(5): 654-682.

IPCC (2012): Managing the risks of extreme events and disasters to advance climate change adaptation. Field, C., Barros, V., Stocker, T., Dahe, Q. (eds).

IPCC (2014): Impacts, Adaption and Vulnerability. Summary for Policymakers. $44 \mathrm{pp}$.

Klösch, M., Habersack, H. (2018): Deriving formulas for an unsteady virtual velocity of bedload tracers EARTH SURF PROC LAND. 2018; 43(7): 1529-1541.

Klösch, M., Bertrand, M., Boz B., Carolli, M., Chouquet, I., Dunst, R., Fragola, G., Gaucher, R., Gmeiner, Ph, Goltara, A., Hauer, C., Holzapfel, P, Langendoen, .E, Javornik, L, Liébault, F., Maldonaldo, E., Marangoni, N., Molnar, P.,
Pessenlehner, S., Pusch, M., Recking, A., Rossi, D., Rozma, D., Santl, S., Stephan, U., Habersack, H. (in Vorbereitung): Technical notes on tools to support planning and design of hydromorphological management and restoration measures. Deliverable D.T2.3.1 of Work Package WPT2, Project HyMoCARES, Interreg Alpine Space Programme, Nr. ASP 445

Knox, J. C. (1983): Response of river systems to Holocene climates; in Late Quaternary Environments of the United States Volume 2, The Holocene (ed.) H.E. Wright, Jr.; University of Minnesota Press, Minneapolis, Minnesota

De Kok, J. L., Grossmann, M. (2010): Large-scale assessment of flood risk and the effects of mitigation measures along the Elbe River. Natural Hazards 52, 143-166.

Krapesch, G., Hauer, C., Habersack, H. (2011): Scale orientated analysis of river width changes due to extreme flood hazards NAT HAZARD EARTH SYS. 2011; 11(8): 2137-2147.

Kreisler, A., Aigner, J., Liedermann, M., Habersack, H. (2014): Geschiebemessung in Österreich. Österreichische Wasser- und Abfallwirtschaft, 66, 297-305; ISSN 0945-358X

Kreisler, A., Moser, M., Aigner, J., Rindler, R., Tritthart, M., Habersack, H. (2017): Analysis and classification of bedload transport events with variable process characteristics GEOMORPHOLOGY. 2017; 291: 57-68.

Lebensministerium (2012): Die österreichische Strategie zur Anpassung an den Klimawandel. Vienna.

Liedermann, M., Gmeiner, P., Kreisler, A., Tritthart, M., Habersack, H. (2018): Insights into bedload transport processes of a large regulated gravel-bed river. EARTH SURF PROCESS LANDF; 43(2): 514-523.

Liedermann, M., Gmeiner, P., Niederreiter, R. Tritthart, M., Habersack, H. (2012): Innovative
Methoden zum Geschiebemonitoring am Beispiel der Donau Österreichische Wasser- und Abfallwirtschaft, 64, 527-534; ISSN 0945-358X Liedermann, M., Tritthart, M., Habersack, H. (2013): Particle path characteristics at the large gravel-bed river Danube: results from a trace study and numerical modelling. EARTH SURF PROCESS LANDF, 38(5), 512-522.

Recking, A. (2012): Influence of sediment supply on mountain streams bedload transport Geomorphology 175:139-150

Rindler, R., Holzapfel, P., Hauer, C., Jury, G., Moser, M., Fischer, A., Gumpinger, C., Habersack, H. (2019): Innovatives Feststoffmanagementkonzept für Wildbacheinzugsgebiete am Beispiel des Strobler Weißenbaches. Österreichische Wasser- und Abfallwirtschaft, 71

Schober, B., Hauer, C., Habersack, H. (2015): A novel assessment of the role of Danube floodplains in flood hazard reduction (FEM-method). Natural Hazards, 2015; 75: S33-S50

Tritthart, M., Glock, K., Glas, M., Yücesan, S. Liedermann, M., Gmeiner, P., Hauer, C., Habersack, $H$. (2019): Erfahrungen in der numerischen Sedimenttransportmodellierung auf unterschiedlichen Skalen - von RANS bis LES. Österreichische Wasser- und Abfallwirtschaft, 71. DOI: 10.1007/s00506-018-0550-0

Turowski, J. M., Rickenmann, D. (2009): Tools and cover effects in bedload transport observations in the Pitzbach. Austria, Earth Surf. Processes Landforms, 34, 26-37, doi:10.1002/esp.1686 Turowski, J. M., Badoux, A., Rickenmann, D. (2011): Start and end of bedload transport in gravel bed rivers. Geophysical Research Letters 38: L04401.

Wolman, M. G. (1954): A method of sampling coarse river-bed material, Eos Trans. AGU 35(6): 951-956. https://doi.org/10.1029/TR035i 006 p00951 